\title{
AN EMERGENCY TOKEN BASED MULTIPLE AC- CESS CONTROL SCHEME FOR WIRELESS ATM NETWORKS
}

\author{
Kun Pang ${ }^{1}$,Zhisheng $\mathrm{Niu}^{1}$,Junli Zheng ${ }^{1}$ and Xuedao $\mathrm{Gu}^{2}$ \\ ${ }^{1}$ Dept. of Electronic Engineering, Tsinghua University, Beijing, 100084,China \\ ${ }^{2}$ Academy of Telecommunication Technology, Beijing, 100083, China \\ \{pk,niu,zhengjl\} @atm.mdc.tsinghua.edu.cn
}

\begin{abstract}
This paper proposes an emergency token based MAC scheme for wireless ATM networks. Token buffer of MT(Mobile Terminal) are constructed in BS(Base Station) for future slot allocation to reduce overhead of access request. The scheduler in base station assigns slots to the sources by polling of token buffer. In order to deal with dynamic nature and stringent QoS of compressed video source, emergency message is defined to inform BS of the source's rate variation and buffer status. At the same time, voice sources are served without reservation. Also, handoff call of voice under micro-cellular environment can be accommodated timely. Emergency token buffer has higher priority over those of VBR and ABR. The emergency token scheduling scheme (ETSS) based on polling of token buffer is effective and easy to implement. Simulations are done to evaluate its performance. We found that the scheduling scheme proposed can significantly improve rtVBR's loss and delay performance while low signalling overhead is needed.
\end{abstract}

Keywords: Wireless ATM, Multiple Access Control, QoS(Quality of Service)

\section{INTRODUCTION}

Wireless communication today has evolved so dramatically that it is highly necessary for wireless system to have more capacity, better quality, and ability to support QoS of multimedia service. As a wireless extension to ATM networks, first proposed in [1], wireless ATM is still a very popular topic nowadays. How to provide wireless access to multimedia services with efficiency and QoS guarantees is one of the most important issues. 
There are some typical MAC(Multiple Access Control) schemes such as DRAMA(Dynamic Resource Allocation Media Access)[2], DQRUMA (Distributed Queueing Request Update Multiple Access)[3], and PNP (Polling with Nonpreemptive Priority)[4]. DRAMA/DQRUMA are proposed to deal with integrated voice and data services, in which VBR services are regarded as data services. In DRAMA scheme, the access of data service is contention based. When contention occurs, the mobile station will retransmit after waiting for random time. DQRUMA is collision free through request access in control frame. These two schemes are well designed to accommodate wireless access, however, there are inherent limitations in them. First, they can not meet the QoS requirements of VBR service. VBR applications (especially real-time video) have relatively strict delay, jitter and loss requirements as well as burst nature over different time-scales. Second, the request access overhead is high. Polling with Nonpreemptive Priority(PNP) is a centralized MAC scheme with passive user. After admitted into the system, the sources will be served by polling. Polling scheme is seldom preferred in wireless system for it will be overburdened by large number of users. In PNP scheme of [4], fixed priorities are assigned to the sources. This implies that lower priority sources may be locked out completely under condition of heavy load [10]. Also, PNP does not consider the dynamic characteristics of video applications as well.

In this paper, we develop a scheduling scheme which can satisfy the dynamic nature involved in wireless multimedia. In stead of dividing the sources into fixed priorities, a dynamic priority scheme is implemented through emergency token. It means that the sources which have the urgent need to transmit will have higher priority. Although our token model based scheduling scheme is to be deployed in TDMA system, it is supposed to adapt to a CDMA one where the bandwidth used in CDMA system can be presented with token as well.

\section{SYSTEM MODEL}

Consider a cellular wireless system, where many mobile stations with multiple connections share a common wireless link. A base station is responsible for access of all mobile stations. It is assumed that user information is accommodated in slots. Since the downlink channel is a broadcasting channel where no contention takes place, the focus of our research is on uplink scheduling scheme in the base station.

It is assumed that each voice call may be modeled as 2-state Markov chain, alternating between $\mathrm{ON}$ and OFF states [11]. Let $N, v_{i}$ and $t_{i+1}$ denote total number of voice connection, number of voice packet arrivals in this frame and 
number of voice talk spurts in next frame. Then the probability for $\mathrm{j}$ talkspurts starting in the next frame, is given by

$$
\operatorname{Pr}\left(t_{i+1}=j\right)=\left(\begin{array}{c}
N-v_{i} \\
j
\end{array}\right)\left(1-p_{\text {off }}\right)^{j} p_{o f f}^{N-v_{i}-j}
$$

It is assumed that slot reordering and reassignment has been implemented to keep all the unused slots at the end of each frame[5]. When a CBR connection enters OFF period, the base station will be informed through a special bit pattern in the last cell of the connection in order to perform slot reordering. In our scheduling scheme, CBR sources are served by periodically feeding token. Therefore, our focus is on the dynamic scheduling of VBR sources. Like the analytical model proposed in [8], both the cell arrival process of nrt-VBR and that of MPEG are assumed to be 2-state MMBP. Though Poisson model isn't quite suitable for modeling MPEG video, MMBP model is exploited to simplify analysis and capture the bursty characteristics.

In order to develop a analytical model, we first assume that both the cell arrival process of nrt-VBR and that of MPEG are 2-state MMBP where the state transition probabilities $\omega_{11}=\omega_{12}=\alpha_{v}$ and $\omega_{11}=\omega_{12}=1-\alpha_{v}(v$ denote the class of VBR cell)[8]. A multi-server dedicated-buffer queueing model $M M B P 1+M M B P 2 / D / s(m 1, m 2)$, where is suitable for the analysis of the proposed MAC scheme.

$\left(N_{1}, N_{2}, Y_{1}, Y_{2}\right)$ is defined as the system state during $n$-th slot, where $N_{1}$ is the sum of the number of occupied slots and the number of C-1(MPEG) cells waiting in $Q_{1}, N_{2}$ is the number of C-2(nrt-VBR) cells waiting in $Q_{2}$, and $Y_{v}$ is the phase of the arrival process $M M B P_{v}$. By using Matrix-Geometric method,[9] the transition probability matrix $P$ is solved and, therefore, the steady-state probability $\operatorname{Pr}\left\{N_{1}=i, N_{2}=j, Y_{1}=l_{1}, Y_{2}=l_{2}\right\}$ is acquired by solving following equation:

$$
\begin{array}{r}
\mathbf{x} P=\mathbf{x} \\
\mathbf{x e}=1
\end{array}
$$

Then the cell loss probability $P_{L v}$ of class $v$ is given by

$$
P_{L v}=\frac{E\left[L_{v}\right]}{E\left[A_{v}\right]}, \quad(v=1,2)
$$




$$
\begin{aligned}
P_{L 1}= & \frac{\sum_{i=0}^{s+m_{1}} \sum_{l_{1}=1}^{r_{1}} E\left[L_{1} \mid N_{1}=i, Y_{1}=l_{1}\right] \operatorname{Pr}\left\{N_{1}=i, Y_{1}=l_{1}\right\}}{\sum_{k_{1}=0}^{\infty} k_{1} \sum_{l_{1}=1}^{r_{1}} a_{1}\left(k_{1}, l_{1}\right) \pi_{1}\left(l_{1}\right)} \\
= & \frac{\sum_{i=0}^{s+m_{1}} \sum_{k_{1}=s_{1}}^{\infty}\left(k_{1}-s_{1}\right)\left\{\sum_{l_{1}=1}^{r_{1}} a_{1}\left(k_{1}, l_{1}\right) \bar{x}_{i l_{1}}\right\}}{\lambda_{1}}
\end{aligned}
$$

As for the loss probability of nrt-VBR traffic, however, it depends not only on the system state but on the parameters of both MPEG and nrt-VBR traffic. Following the similar probability arguments, we have

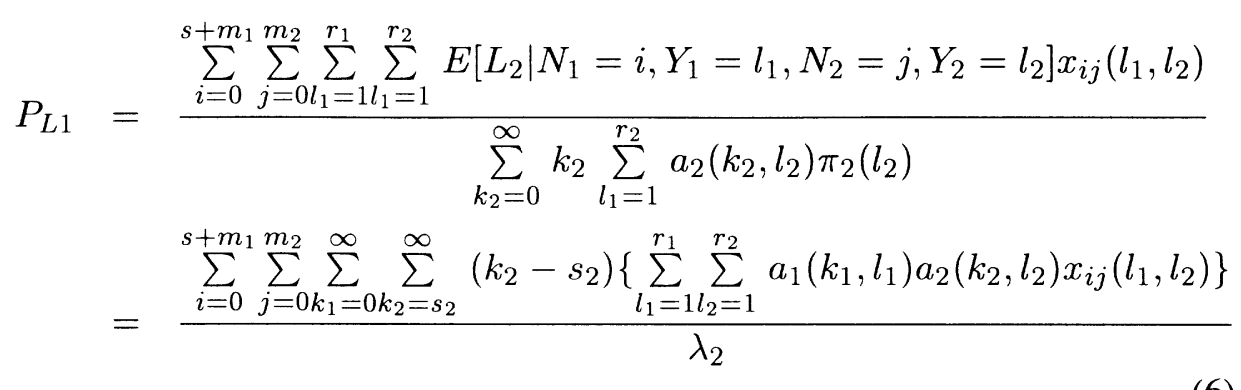

Since the regulated nrt-VBR's delay bound under PGPS service discipline, $D_{i}^{*} \leq \frac{\sigma}{\rho}$, is quite tolerable for bursty service[7]. We choose large buffer size for nrt-VBR regulated by leaky $\operatorname{bucket}\left(\rho_{j}, \sigma_{j}\right)$. To adapt to rt-VBR's dynamic characteristics and delay requirement, nrt-VBR is handled as class-2 traffic. However, the priority is a dynamic priority that benefits both fairness and efficiency.

\section{FRAME STRUCTURE}

We emphasize our research on slot allocation scheme of uplink channel. A TDMA based frame structure is shown in Figure 1 . The length of MAC frame is $T$. The control part of a frame is consisted of emergency minislots where emergency messages are transmitted through S_ALOHA and request minislots where access requests are made. The data part of a frame is consisted of data slots where user information packetized in wireless ATM cells are transmitted.. Our MAC structure is somewhat like that proposed in [8], however, reservation 


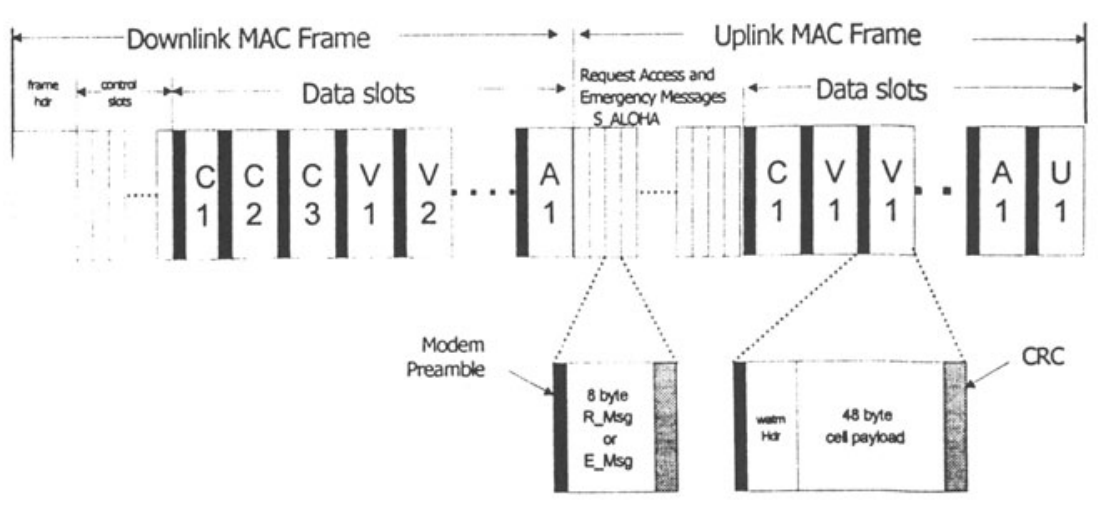

Figure 1 TDMA based MAC frame structure

slots is unneccessarry any more and MAC frame is not divided fixedly into CBR, VBR subframe to accommodate multi-rate CBR service. Also, we distinguish Emergency_Message from Access_Request in the control part of MAC frame, which is based on the idea that system will be overloaded when there are many emergency messages.

There are three types of 8-byte Emergency_Message defined: a)MPEG frame rate variation is over a certain threshold: $M P G_{-}$Scene_Message (We have known that VBR video is known to exhibit multiple time scale variations. Between scenes there is a significant change in the bit rate. For this reason, MPEG rate variation is emphasized in source modeling for accuracy.); b)rt-VBR source buffer is over threshold: VBR_Threshold_Message; c)CBR source enter ON period: $C B R_{-}$Contend_Message. After receiving an emergency message, BS will put emergency tokens into emergency token buffer following the rule proposed in next section.

\section{EMERGENCY TOKEN BASED SCHEDULING SCHEME(ETSS)}

In wireless ATM scenario, the traffic sources send traffic descriptor and specified QoS to BS during connection setup period. After admitting access of sources, the scheduler in the base station will allocate slots to the connections according to the tokens got from token buffer where the tokens are generated with certain rule. The token polling shown in Figure 2 seems like mathematical 
model of reservation but with more flexibility. The token is generated with following rules:

i) For CBR source $j$ with rate $\lambda_{j}$, its token is also generated with $\lambda_{j}$;

ii) For nrt-VBR source $j$ regulated by leaky bucket $\left(\rho_{j}, \sigma_{j}\right)$, its token is generated with sustained cell rate $\rho_{j}$;

iii) For MPEG-VBR source $j$, its token is generated variably according its frame rate $\rho_{I, j}, \rho_{P, j}, \rho_{B, j}$.

iv) For ABR service with specified MCR rate $\eta_{j}$, its token is generated with MCR rate $\eta_{j}$ after $t_{a}$ ms to meet its Poisson characteristics.

In uplink slot allocation, the scheduler polls the token buffers and assigns slots to the sources identified by tokens. If any Emergency_Message is received, the scheduler will first check out message type and change models for sources as following:

1) If it is a $C B R \_C o n t e n d \_M e s s a g e$, put one token containing the connectionID HOL into emergency buffer, then start generate token of the corresponding source into CBR token buffer. Therefore it doesn't impact other CBR's CDV.

2) If it is a $M P G_{-} S_{\text {Scene_Message, change token generating rate in model }}$ and put $N_{j}^{i+1}$ emergency tokens into emergency buffer. Take statistical multiplexing gain into account, scheduler will stop sending $N_{j}^{i+1}$ emergency tokens after a length of MPEG frame.

$$
N_{j}^{i+1}=\Delta R_{\text {frame_change }} * T
$$

In equation above, if $\Delta R_{\text {frame_change }} \leq 0$, then $N_{j}^{i+1}=0$, only token generation rate is changed.

3) If it is a $V B R \_T h r e s h o l d \_M e s s a g e$, put $N_{\text {buffer_length }}$ emergency tokens into emergency buffer. For sake of statistical multiplexing, stop sending token for $t=N_{\text {buffer_length }} / \rho_{j} \mathrm{~s}$ after burst length.

When slot allocation begins, the scheduler scans the token buffer and assigns slots.

1) The scheduler scans CBR token buffer periodically. Any token found is removed and one slot is assigned to the corresponding CBR source. 


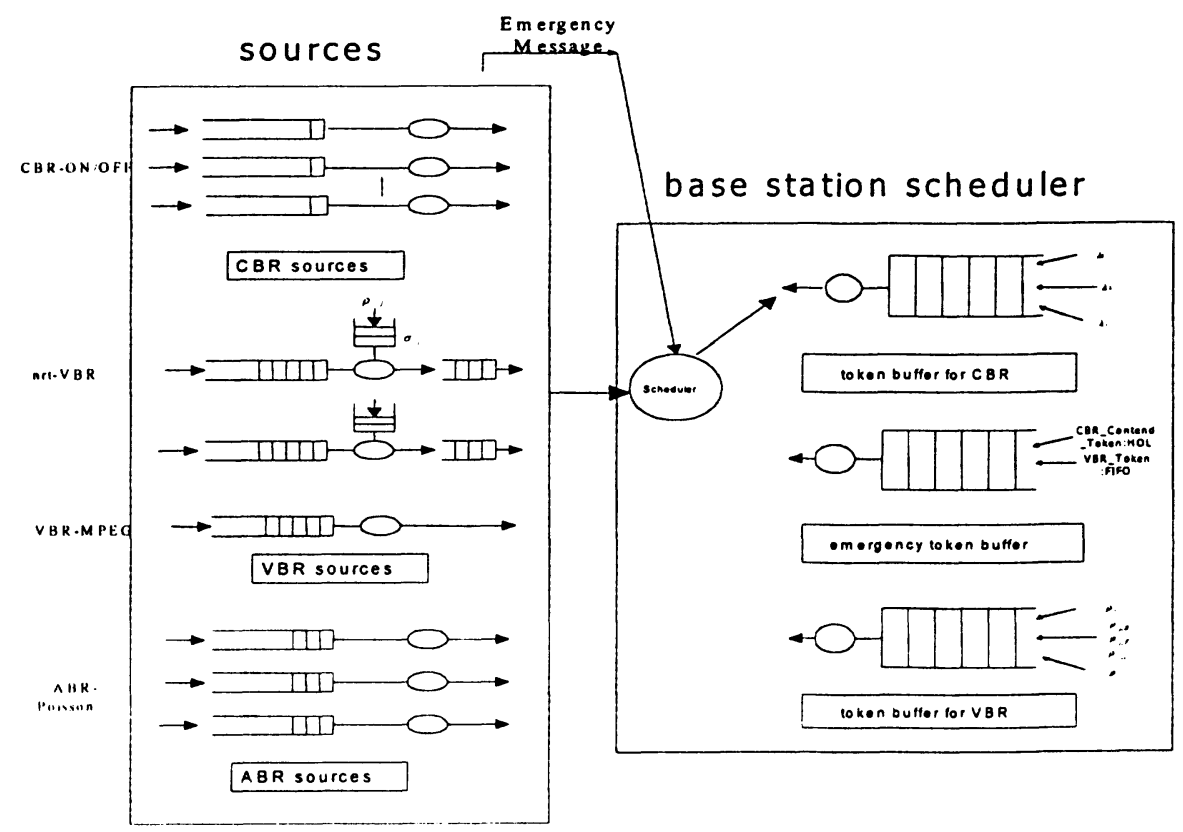

Figure 2 Dynamic token model based sheduling scheme

2) In order not to affect CBR's jitter requirement, emergency slot allocation is after CBR token buffer polling cycle. If there is no token found in CBR token buffer, the scheduler scans EMERGENCY token buffer for token and assigns slots to corresponding sources.

3) When there is no token in the emergency tokens, the scheduler will enter VBR token polling cycle. The scheduler scans the VBR token buffer and assigns slots correspondingly until there is no token for VBR sources. The extraction of tokens is exhaustive so that slot allocation is continuous except that it will be interrupted by periodical CBR token polling.

4) After slot allocation for CBR and VBR is finished, the scheduler will assign remaining slots in a MAC frame to $A B R$ and UBR sources with fair and efficient sharing.

\section{SIMULATION RESULTS}

It is assumed that link speed is $10 \mathrm{Mbps}$ in our system. The MAC subframe duration $T$ is $2 \mathrm{~ms}$ for delay and jitter requirements of CBR traffic. MPEG 
source's frame rate is $25 \mathrm{~Hz}$, therefore, video frame length $(40 \mathrm{~ms})$ is sufficiently lager than $T_{s}$ so that information of video frame change is much meaningful. MPEG-I cells are generated because of limited wireless bandwidth, where average bit rate is from 0.8 to $1.6 \mathrm{Mbps}$ and peak-to-average ratio is 4 . As for the ON/OFF parameter of voice will depend on coding technique. We use the parameters proposed in [11]. As for general VBR service, Poissonian model is enough for simulation. And gamma beta autoregressive process (GBAR) proposed in [6] was used to generate MPEG video source traces in our simulation, which is considered as an appropriate model for VBR video source.

We write a MAC simulator in $\mathrm{C} / \mathrm{C}++$ to verify our scheduling schemeETSS and an error-free environment is assumed. The simulator is consisted of several modules of aloha manager, sources, slot allocation and token manager, etc. Since it is in microcellular environment, transmitting delay is ignored and only queueing delay is considered. From results in Figure 3, it is shown that rt-VBR's CLP(Cell Loss Probabilities) performance is improved under ETSS more than that of VBR round-robin. Emergency token may assign priority to corresponding source dynamically so that QoS of realtime and bursty source can be satisfied. The buffer size $c$ can be expressed as the time required to empty a full buffer(buffer drain time). Buffer drain time $B_{i}=c / d_{i}$, where $d_{i}$ is service rate of queue $i$. In Figure 4, we compare simulation results with analytical results in [8] of two kind of cells. As we expected, the performance curves of MPEG cells accord with each other under light MPEG load. But performance will deteriorate under heavy load. The reason is S_ALOHA transmission of emergency message will degrade greatly accompanied with emergency load's increase. The average queueing delay of MPEG cells in Figure 5 increasing drastically under heavy load is also due to the reason above. Number of emergency slots is the essential parameter of our model. From Figure 6, we can find that performance improvement is high when minislot number varies from 5 to 10(infinite buffer is used here). But improvement is rather low with the minislot number increased because system will be overloaded by emergency handling. And we can see that the scheduling scheme can work up to high utilization of $90 \%$ for the scheme exploits VBR's statistical multiplexing efficiently, however, the utilization is supposed to fall under error link, where retransmission is frequent. 


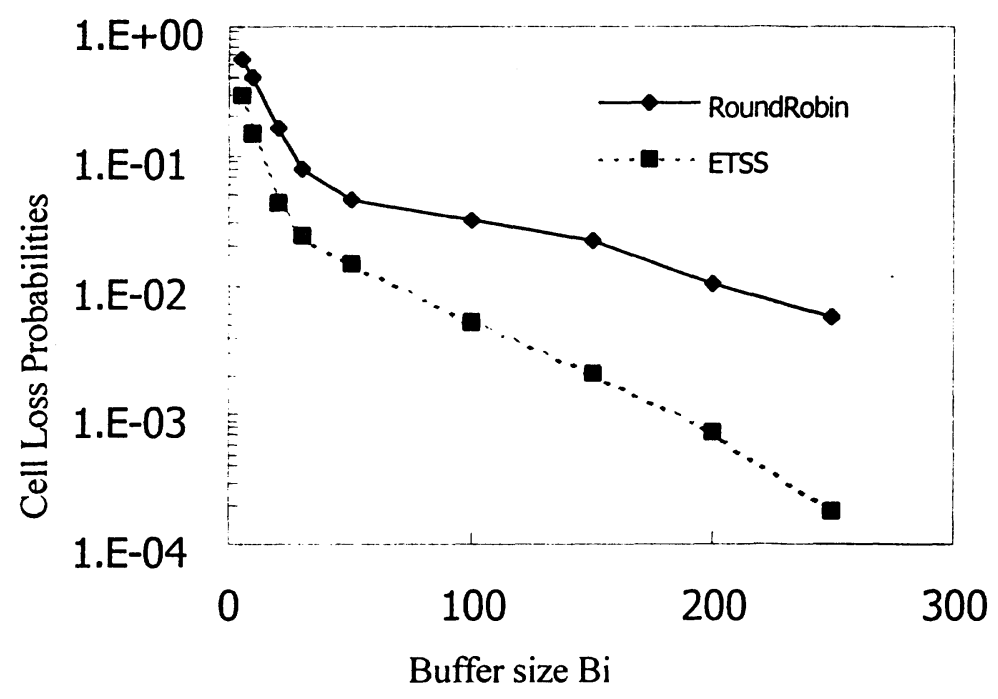

Figure 3 Buffer size vs cell loss probabilities (with utilization of $60 \%$, Number of emergency slots $=10$ )

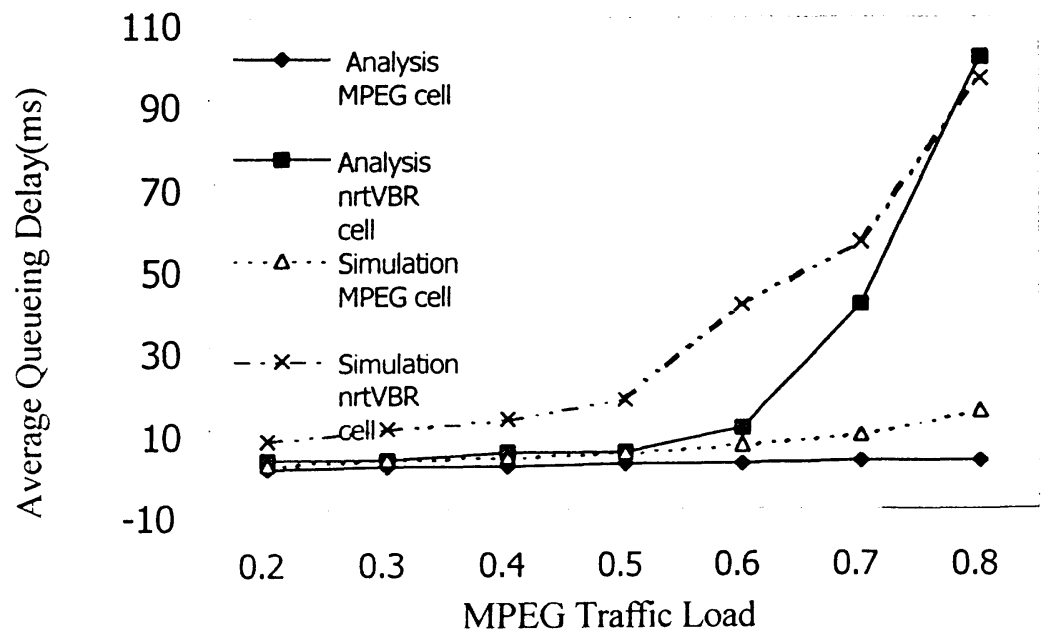

Figure 4 MPEG traffic load vs cell loss probabilities (with utilization of $60 \%$, Number of emergency slots $=10$ ) 


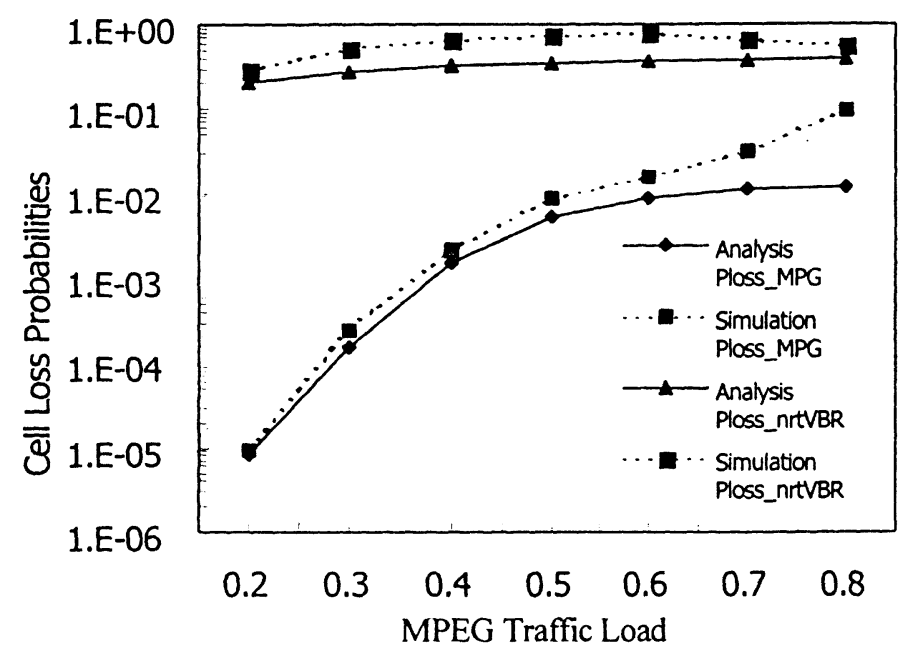

Figure 5 MPEG traffic load vs average queueing delay (with utilization of $60 \%$, Number of emergency slots $=10$ )

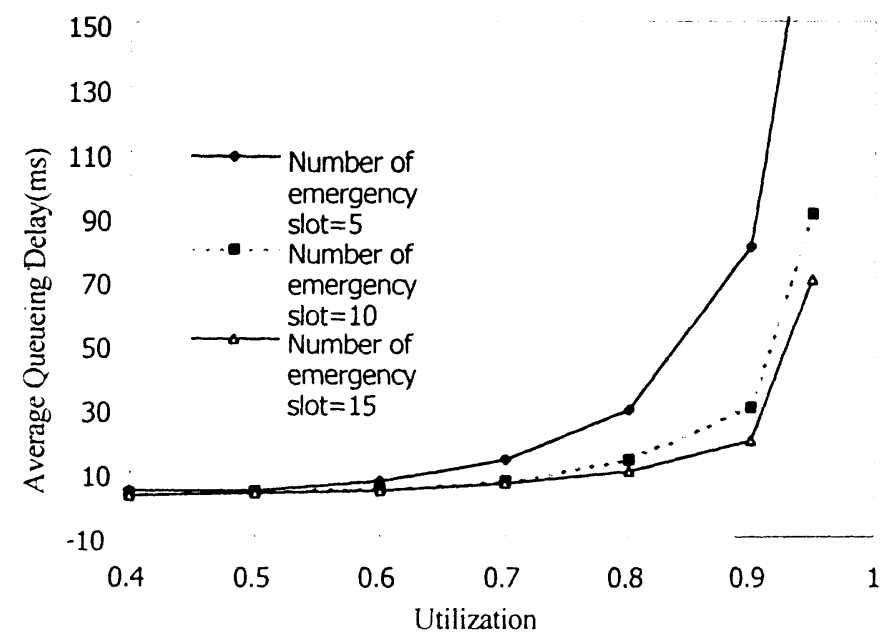

Figure 6 Average Queueing Delay of MPEG cell vs Utilization (60\%MPEG load of VBR load) 


\section{CONCLUSION}

A new multiple access protocol based on combination of source modeling and dynamic scheduling is developed. The performance of the new scheme has been evaluated by simulation. The results show that the delay and loss performance of dynamic MPEG sources can be improved greatly in designing wireless multiple access protocol. The future work is the combination of our scheduling scheme with a modified CAC strategy to meet QoS of multiple handoff connections.

\section{Acknowledgments}

This work is partially supported by Fujitsu Laboratory Ltd.

\section{References}

[1] D. Raychaudhuri and N.D. Wilson, ATM-based transport architecture for multiservices wireless personal communication networks, IEEE JSAC12 (1994), pp.1401-1414.

[2] B. A. Akyol D.C. Cox "A Dynamic Resource Allocation and Media Access Control Proposal for a Multi-tier Wireless ATM Network", Technical Report, Stanford University, June 1997.

[3] M. J. Karol, Z. Liu and K. Eng, "Distributed-queueing Request Update Multiple Access (DQRUMA) for Wireless Packet (ATM) Networks", IEEE International Conference on Communications (ICC'95), Seattle, USA. June 1995, pp.1224-1231.

[4] C-S. Chang "Guaranteed Quality-of-Service Wireless Access to ATM Networks", IEEE J. on Selected Areas in Communications, Vol.15, No 1, January 1997, pp.106 - 117.

[5] Xiaoxin Qiu, Victor O.K.Li, "A multiple access scheme for multimedia traffic in wireless ATM", IEEE/ACM Mobile Networks and Applications. Jan 1996, pp. 259-272

[6] Daniel P. Heyman, "The GBAR Source Model for VBR Videoconferences", IEEE/ACM Transactions on Networking, vol. 5. No. 4, August 1997, pp. 554-560.

[7] M. Schwartz, "Broadband Integrated Networks" Prentice Hall Inc.1996, pp.254-261. 
[8] Z. Niu and KUBOTA "An Adaptive MAC Scheme for Wireless ATM and its Performance Evaluation", Chinese Journal of Electronics, Vol.7, No.4, 1998, pp341-348.

[9] M. F. Neuts, "Matrix-Geometric Solutions in stochastic Models" The John Hopkins University Press.1981, pp.254-261.

[10] A.S.Tanenbaum, "Computer Networks". EngleWood Cliffs, NJ: PrenticeHall, 1981.

[11] S.Q.Li, 'Dynamic Bandwidth Allocation on a Slotted ring with Integrated services". IEEE Transactions on Communication, Vol 36, No.7, pp.827828. 